\title{
Use of thermokinetic EMF and electrical resistance for quality control of elongated products made of shape memory alloy
}

\author{
O. A. Petrova-Burkina ${ }^{\dagger}$, V. V. Rubanik Jr., V.V. Rubanik \\ †olchik.1987@mail.ru \\ Institute of Technical Acoustics of NAS of Belarus, 13 Generala Lyudnikova Av., Vitebsk, 210009, Belarus
}

\begin{abstract}
The behavior of the thermokinetic EMF and electrical resistance upon nonstationary heating of the elongated TiNi wire samples with a near-equiatomic composition having sections subjected to elastic and plastic deformation was studied. It was found that the thermokinetic EMF value sharply increases in the deformed zone in the $1^{\text {st }}$ thermal cycle with the movement of the heating zone along the sample. The increase in the relative deformation from 1 to $30 \%$ leads to a change in thermokinetic EMF $(|\Delta E|)$ from 0.01 to $0.37 \mathrm{mV}$. If the sample undergoes deformation up to $2 \%$, the thermokinetic EMF value in the deformation area corresponds to the value on the nondeformed section during the $2^{\text {nd }}$ thermal cycle. The value of $|\Delta E|$ is increased by $0.1 \mathrm{mV}$ during deformation from 2 to $10 \%$ and is not changed with an increase in deformation up to $30 \%$. The behavior of the electrical resistance is similar to the behavior of the thermokinetic EMF when the heating zone moves along the length of the Ti-50 at.\% Ni wire sample in deformed zone from 2 to $15 \%$. The electrical resistance increases sharply upon the $1^{\text {st }}$ thermal cycle in the deformation zone. The electrical resistance increases by $25 \mu \Omega \cdot \mathrm{cm}$ with an increase in the applied deformation up to $15 \%$. The value of electrical resistance does not change in the zone of deformation up to $2 \%$ upon the $2^{\text {nd }}$ thermal cycle. When the value of applied deformation falls in the range from 5 to $15 \%$, the electrical resistance falls by $5 \div 20 \mu \Omega \cdot \mathrm{cm}$. Deformation of the Ti-50 at.\% Ni sample leads to a change in the properties of the alloy in the deformation zone, causing a shift in the characteristic temperatures of the phase transition and a change in the thermokinetic EMF and electrical resistance when the heating zone passes through the deformation zone. Changes in the thermokinetic EMF and electrical resistance as the heating region passes through the deformation zone are associated with a change in the characteristic temperatures of the phase transition. Based on the experimental data, a method and devices for determining inhomogeneous areas in elongated products made of shape memory alloys were developed. The method allows the value of thermokinetic EMF or electrical resistance to be continuously recorded during winding the wire when its section is heated above the temperature of the reverse phase transition. Tracking the change in the thermokinetic EMF or electrical resistance, it is possible to determine the sections of the material, which differ in physical properties from the predeterminated properties.
\end{abstract}

Keywords: titanium nickelide, thermokinetic EMF, reverse phase transition, deformation, quality control.

УДК: 53.096

\section{Использование термокинетической ЭДС и электросопротивления для контроля качества протяженных изделий из сплава с памятью формы}

\author{
Петрова-Буркина О. А. ${ }^{\dagger}$ Рубаник В. В. мл., Рубаник В.В. \\ Институт технической акустики НАН Беларуси, пр. Генерала Людникова, 13, Витебск, 210009, Беларусь
}

Исследовано поведение термокинетической ЭДС и электросопротивления при нестационарном нагреве протяженных проволочных образцов TiNi состава близкого к эквиатомному, содержащих участки, подвергнутые упругой и пластической деформации. Установлено, что в 1-ом термоцикле в процессе перемещения области нагрева вдоль образца, на участке деформации резко возрастает величина термокинетической ЭДС. Увеличение относительной деформации от 1 до 30\% приводит к изменению термокинетической ЭДС $(|\Delta E|)$ от 0.01 до 0.37 мВ. Во 2-ом термоцикле, если образец испытывал деформацию до 2\%, величина термокинетической ЭДС в области деформации 
соответствует значению на недеформированных участках. При деформации от 2 до $10 \%$ значение $|\Delta E|$ увеличивается до $0.1 \mathrm{mB}$ и при увеличении деформации до 30\% не изменяется. Характер поведения электросопротивления при перемещении области нагрева по длине проволочного образца Тi-50 ат.\% Ni в зоне деформации от 2 до $15 \%$ схож с поведением термокинетической ЭДС. В 1-ом термоцикле на участке деформации величина электросопротивления резко возрастает. С ростом деформации до $15 \%$ электросопротивление увеличивается на 25 мкОм·см. Во 2-ом термоцикле в зоне деформации до $2 \%$ величина электросопротивления не изменяется. В остальных случаях — падает. При деформации от 5 до 15\% электросопротивление уменьшается на $5 \div 20$ мкОм·см. Деформирование образца Ti-50 ат.\% Ni приводит к изменению свойств сплава, что влечет за собой смещение характеристических температур фазового перехода и изменение термокинетической ЭДС и электросопротивления при прохождении области нагрева через зону деформации. На основе полученных экспериментальных данных разработаны метод и устройства определения неоднородных участков протяженных изделий из сплавов с памятью формы. Метод заключается в непрерывной регистрации при перемотке проволоки значений термокинетической ЭДС или электросопротивления при нагреве ее участка выше температуры обратного фазового перехода. По изменению термокинетической ЭДС или электросопротивления определяют участки материала, которые отличаются физическими свойствами от заданных.

Ключевые слова: никелид титана, термокинетическая ЭДС, обратный фазовый переход, деформирование, контроль качества.

\section{1. Введение}

В настоящее время широкое практическое применение в различных отраслях науки и техники находят сплавы с термоупругими фазовыми превращениями, в том числе на основе титана и никеля [1-4]. Важными характеристиками таких сплавов являются температуры мартенситных переходов, при которых происходит формовосстановление элементов с памятью формы. На характеристические температуры влияет как соотношение основных компонентов (титана и никеля), так и величина деформации данных сплавов (в результате появления дислокаций, фазового и деформационного наклепа и т.д.) [5]. Известно [6], что механические свойства материалов с термоупругими мартенситными превращениями существенным образом зависят от температуры деформирования, точнее, её расположения относительно характеристических температур обратимых фазовых переходов. Таким образом, исследование влияния деформации на характеристические температуры фазовых переходов никелида титана является важным этапом на пути внедрения этого материала в промышленность.

Ранее авторами установлено [7-10], что при движении области нагрева вдоль проволочного образца никелида титана состава близкого к эквиатомному (при условии протекания в области нагрева обратного фазового перехода), в связи с изменением электронных свойств сплава за счет термоупругого фазового превращения, возникает постоянная по величине и направлению термокинетическая ЭДС, которая составляет до 0.22 мВ. Термоциклирование таких образцов приводит к падению величины термокинетической ЭДС. Также установлено [11], что движение границы раздела фаз в образцах никелида титана при нестационарном нагреве вызывает изменение электросопротивления. Термоциклирование образца в температурном интервале обратного-прямого мартенситного превращения за счет нестационарного нагрева приводит к изменению значений электросопротивления как в высокотемпературной В2, так и в мартенситной В19'-фазе.
Целью данной работы являлось исследование поведения термокинетической ЭДС и электросопротивления при нестационарном нагреве протяженных проволочных образцов TiNi состава близкого к эквиатомному, содержащих участки, подвергнутые упругой и пластической деформации, а также на основе полученных данных разработка методов и устройств контроля однородности физико-механических свойств протяженных, например проволочных, TiNi изделий.

\section{2. Оборудование и методика исследования}

Исследовали проволочные образцы никелида титана состава близкого к эквиатомному, изготовленные в промышленном центре ООО «Промышленный центр МАТЭК-СПФ», длиной 40 см, диаметром 0.6 мм, предварительно отожжённые при температуре $700^{\circ} \mathrm{C}$ в течение 30 минут с закалкой на воздухе.

Кинетику мартенситных превращений исследовали методом дифференциальной сканирующей калориметрии (ДСК) на калориметре DSC822e (METTLER TOLEDO) при скоростях нагрева и охлаждения $10^{\circ} \mathrm{C} / \mathrm{Mин}$. По калориметрическим кривым определяли температуры начала $\left(A_{\text {н }}, M_{\text {н }}\right)$ и окончания $\left(A_{\mathrm{K}}, M_{\mathrm{K}}\right)$ мартенситных переходов методом пересечения касательных по стандарту ASTM F2004-00 [12]. В исходном состоянии образцы находились в мартенситном состоянии, характеристические температуры сплава: $M_{\mathrm{H}}=45^{\circ} \mathrm{C}, M_{\mathrm{K}}=33^{\circ} \mathrm{C}$, $A_{\mathrm{H}}=57^{\circ} \mathrm{C}, A_{\mathrm{K}}=78^{\circ} \mathrm{C}$, реализация фазовых переходов осуществлялась по схеме $\mathrm{B} 2 \leftrightarrow \mathrm{B} 19^{\prime}$.

Термокинетическую ЭДС и электросопротивление измеряли прямым способом цифровым милливольтметром МНИПИ В7-72. Испытания проводили на экспериментальной установке, конструкция которой позволяет перемещать область нагрева по образцу с заданной скоростью. Места контакта образца с измерительными медными проводами термоизолировали. Температуру на поверхности образца контролировали тепловизором с точностью $\pm 2{ }^{\circ} \mathrm{C}$. Предварительно производили чернение поверхности проволоки. Рабочая длина образцов составляла 30 см. В процессе измерений скорость пере- 
мещения области нагрева была постоянной и составляла $0.4 \mathrm{~cm} / \mathrm{c}$, максимальная температура проволоки в области нагрева $150^{\circ} \mathrm{C}$, что выше температуры окончания обратного фазового перехода в изучаемом материале.

Деформирование одноосным растяжением участков проволочных образцов Тi-50 ат.\% Ni проводили на испытательной машине ИП 5158-5 со скоростью 30 мм/мин при комнатной температуре $\left(23 \pm 1^{\circ} \mathrm{C}\right)$, когда материал находился в мартенситном состоянии. Прямолинейный образец TiNi крепили в захваты разрывной машины. Расстояние между зажимами соответствовало зоне деформации $\sim 5$ см. Для исключения деформирования проволоки в зажимах захвата образец помещался между двумя деревянными пластинами. Растяжение образцов в мартенситной фазе сопровождалось переориентацией мартенситных пластин при напряжениях 250 МПа, предел текучести составлял 500 МПа.

\section{3. Результаты и их обсуждение}

Установлено, что в сплаве никелида титана эквиатомного состава значение термокинетической ЭДС зависит от заданной предварительной деформации. При величине относительной деформации $\varepsilon \approx 11 \%$ в 1-ом термоцикле в процессе движении области нагрева вдоль образца, в зоне деформации, резко возрастает величина термокинетической ЭДС на 0.26 мВ (Рис. 1). В последующих 2-ом и 3-ем термоциклах на участке деформации ее величина падает на 0.1 мВ.

Зависимости изменения термокинетической ЭДС $(|\Delta E|)$ в зоне деформирования от величины относительной деформации растяжением (ع) показывают (Рис. 2), что в 1-ом термоцикле увеличение относительной деформации с 1 до 30\%, приводит к росту термокинетической ЭДС на $0.01 \div 0.37$ мВ. Во 2-ом термоцикле величина изменения термокинетической ЭДС зависит от значения первоначально заданной относительной деформации. Если образец испытывал деформацию до 2\%, то термокинетической ЭДС возвращается к первоначальному значению $0.22 \mathrm{mB}$, т.е. ее значение остается одинаково на всех участках образца. При деформировании образца от 2 до 10\%, когда в материале происходит переориентация мартенситных пластин, величина изменения термокинетической ЭДС $(|\Delta E|)$ составляет $0.02 \div 0.1$ мВ. При дальнейшем увеличение относительной деформации до $30 \%$, когда в образце происходит пластическое деформирование мартенситной фазы, величина изменения термокинетической ЭДС $(|\Delta E|)$ остается постоянной порядка $0.1 \mathrm{mB}$.

Установлено, что характер поведения электросопротивления при перемещении области нагрева по длине проволочного образца Тi-50ат.\% $\mathrm{Ni}$ после деформирования участка образца одноосным растяжением (Рис. 3) при значении относительной деформации от 2 до 15\% схож с поведением термокинетической ЭДС. В 1-ом термоцикле (Рис. 3а) в зоне деформации образца величина электросопротивления резко возрастает на $2 \div 25$ мкОм · см. Чем больше задана деформация, тем на большую величину увеличивается электросоп-

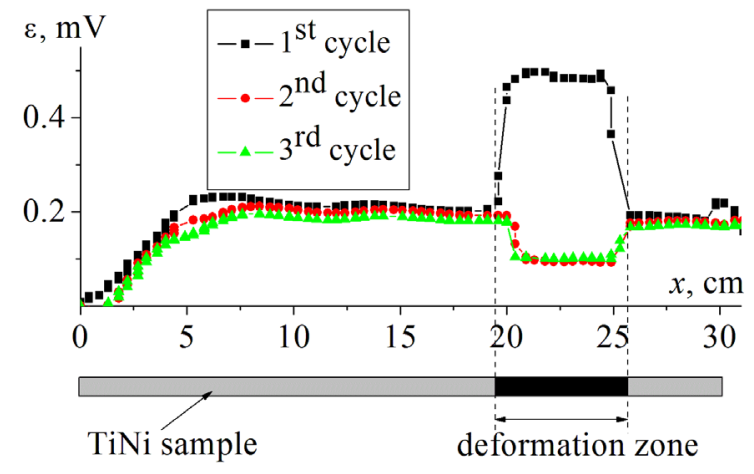

Puc. 1. (Color online) Изменение термокинетической ЭДС от местоположения области нагрева образца Ті-50ат.\% Ni (в зоне деформации $\varepsilon \approx 11 \%$ ).

Fig. 1. (Color online) Dependence of the thermokinetic EMF value on the location of the heating zone of the Ti-50 at.\% Ni sample (on the deformation zone $\varepsilon \approx 11 \%$ ).

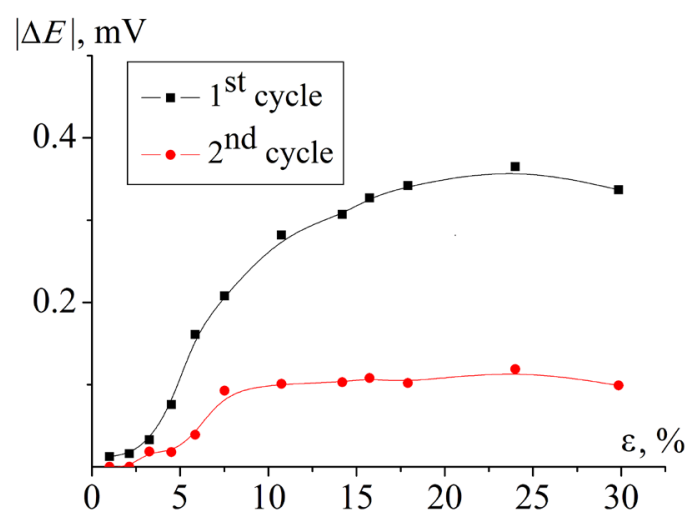

Puc. 2. (Color online) Зависимость изменения термокинетической ЭДС от величины относительной деформации растяжением в образце Тi-50 ат.\% $\mathrm{Ni}$.

Fig. 2. (Color online) Dependence of the change in thermokinetic EMF on the magnitude with respect to tensile deformation in the Ti-50 at.\% Ni sample.

ротивление. Во 2-ом термоцикле (Рис. 3 b) в зоне предварительной деформации до $2 \%$ величина электросопротивления не изменяется. В остальных случаях - падает. При предварительной деформации от 5 до 15\% электросопротивление уменьшается на $5 \div 20 \mathrm{мкOм} \cdot \mathrm{cm}$.

Как известно $[1,5,13,14]$, в результате деформирования никелида титана возникают различного рода дефекты (дислокации, мартенситный наклеп и т.д.), которые в свою очередь влияют на термоэлектрические характеристики материала. В нашем случае (Рис. 4), в 1-ом термоцикле деформация образцов Ті-50 ат.\% Ni приводит к смещению температурного интервала обратного фазового перехода (нагрев) в сторону более высоких температур. Увеличение относительной деформации до $30 \%$ приводит к росту температур обратного фазового перехода на $50^{\circ} \mathrm{C}$. Также, согласно калориметрическим кривым, деформирование образцов Тi-50 ат.\% Ni от 11 до $30 \%$ влияет на кинетику фазового перехода при нагреве, переход осуществляется по двухстадийной схеме.

Во 2-ом цикле, если образец был деформирован до $2 \%$, происходит возврат температур фазового пере- 


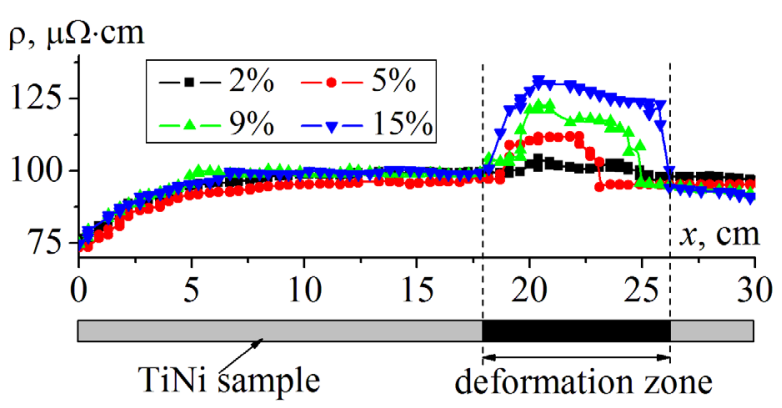

a

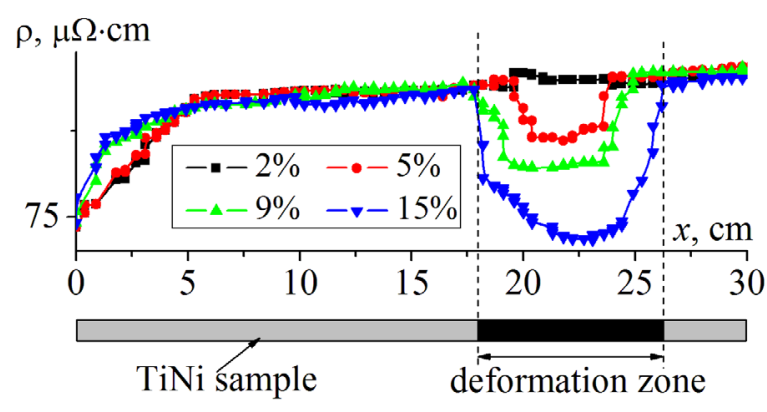

b

Pиc. 3. (Color online) Изменение электросопротивления от местоположения области нагрева образца Тi-50 ат.\% Ni для 1-го (a) и 2-го термоцикла (b) (зона деформации указана для $\varepsilon \approx 15 \%$ ).

Fig. 3. (Color online) Dependence of electrical resistance on the location of the heating region of theTi-50at.\%Ni sample for the $1^{\text {st }}$ (a) and $2^{\text {nd }}$ thermal cycle (b) (deformation zone indicated for $\varepsilon \approx 15 \%$ ).

хода к первоначальным значениям, т.е. как в недеформированном образце. При предварительном деформировании от 2 до 10\% температуры обратного фазового перехода уменьшаются на $5 \div 10^{\circ} \mathrm{C}$. Дальнейшее увеличение предварительной деформации до 30\% незначительно влияет на температуры обратного фазового перехода. При этом, согласно калориметрическим кривым, характеристические температуры прямого фазового перехода в интервале степеней деформации $2 \div 30 \%$ не изменяются, т. е. остаются такими, как в 1-ом термоцикле.

Таким образом, изменения термокинетической ЭДС и электросопротивления при прохождении области нагрева через зону деформации связаны с изменением характеристических температур фазового перехода. Возникновение термокинетической ЭДС обусловлено наличием контактной разности потенциалов между нагретым и ненагретыми участками $\mathrm{TiNi}$, находящимися в разных фазовых состояниях $[7,9,15,16]$. В 1-ом термоцикле, при перемещении участка нагрева через зону деформации происходит восстановление формы образца, т.е. инициирование эффекта памяти формы в материале. Возникает еще одна составляющая термокинетической ЭДС, связанная с возникновением разности потенциалов на границах деформированной зоны в результате изменения температур фазового перехода на деформированном и недеформированном участке проволочного TiNi образца. Все это приводит к росту термокинетической ЭДС и электросопротивления в 1-ом цикле нагрева.

Во 2-ом термоцикле, при предварительной деформации до $2 \%$, т.е. в упругой области, температуры фазового перехода в зоне деформации такие же, как температуры на остальных участках образца. Естественно, термокинетическая ЭДС и электросопротивление остаются в этом случае одинаковыми на всех участках образца. При предварительной деформации более $2 \%$, температуры фазового перехода сначала уменьшаются, затем стабилизируются по своему значению. При этом температура окончания прямого фазового перехода становится ниже комнатной, т.е. материал при охлаждении не полностью переходит в мартенситное состояние. Все это, в отличие от 1-ого термоцикла, приводит к уменьшению и дальнейшей стабилизации значений термокинетической ЭДС и электросопротивления в зоне деформации. Эти результаты

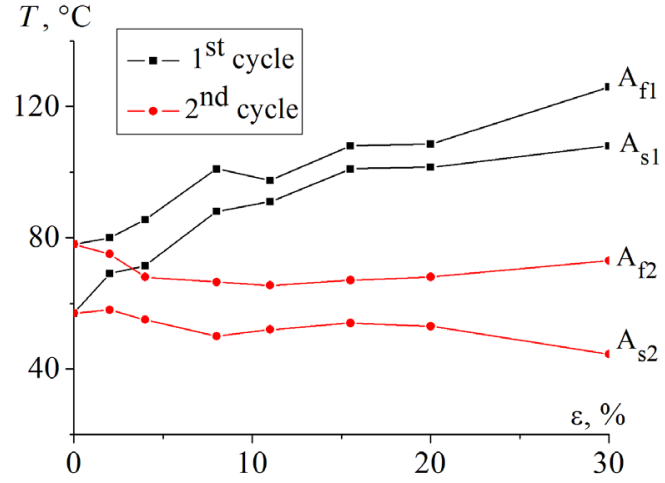

Pис. 4. (Color online) Зависимость характеристических температур фазовых переходов образцов Ti-50 ат.\% Ni от деформации.

Fig. 4. (Color online) Dependence of the characteristic temperatures of phase transitions of the $\mathrm{Ti}-50$ at. $\% \mathrm{Ni}$ samples on deformation.

согласуются с данными работ $[7,9,10,11]$ для термоциклирования образцов Ti-50 ат.\% Ni. Однако физика данного процесса не совсем понятна и объяснима и требует дальнейших исследований.

В связи с тем, что термокинетическая ЭДС и электросопротивление характеризуют дефектную структуру сплава, их можно использовать в качестве инструмента для контроля качества протяженного изделия из сплава с памятью формы. Исходя из вышесказанного, предложены метод и устройство определения неоднородных участков протяженных изделий из сплавов с памятью формы [17,18].

Метод заключается в непрерывной регистрации в процессе перемотки проволоки значений термокинетической ЭДС или электросопротивления при нагреве ее участка выше температур обратного фазового перехода. По изменению термокинетической ЭДС или электросопротивления определяют участки материала, которые отличаются физическими свойствами и подлежат выбраковке.

Устройство (Рис. 5) работает следующим образом: изделие 6 сматывают с подающего блока 1 и наматывают на принимающий блок 7. Между блоками расположена термостатная камера 4, в которой поддерживается постоянной заданная температура с помощью известных 


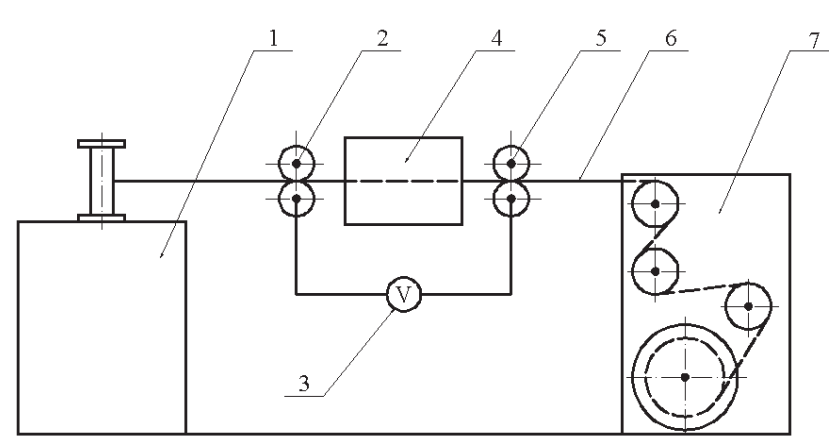

Рис. 5. Устройство контроля качества протяженного изделия из сплава с памятью формы: 1,7 - принимающие блоки; 2, 5 - роликовые контакты; 3 - вольтметр (омметр); 4 - термостатная камера; 6 - изделие (в нашем случае это проволока).

Fig. 5. The quality control device for an elongated shape memory alloy product: $1,7-$ receiving blocks; $2,5-$ roller contacts; 3 - voltmeter (ohmer); 4 - thermostat chamber; 6 - product (in our case, it is a wire sample).

систем регулирования. Два роликовых контакта 2 и 5 для измерения термоЭДС (электросопротивления) подключают к изделию, соответственно, на входе и выходе из термостатной камеры 4, где изделие находится при одинаковой температуре, меньшей температуры

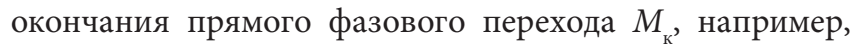
комнатной. ТермоЭДС (электросопротивление) непрерывно регистрируют с помощью вольтметра 3 в режиме реального времени.

\section{4. Заключение}

Установлено, что в сплаве никелида титана состава близкого к эквиатомному на значения термокинетической ЭДС и электросопротивления при нестационарном нагреве влияет наличие в образце деформационных участков. При этом, в 1-ом термоцикле в зоне деформации, термокинетическая ЭДС и электросопротивление возрастают на $0.01 \div 0.37$ мВ и $2 \div 25$ мкОм.см. В следующем термоцикле, в случае если предварительная деформация составляла более $2 \%$, на участке деформации их величина уменьшается на $0.02 \div 0.1 \mathrm{mB}$ и $5 \div 20$ мкОм·см. Деформирование образца Ті-50 ат.\% Ni приводит к изменению свойств сплава, что влечет за собой смещение характеристических температур фазового перехода и изменение термокинетической ЭДС и электросопротивления при прохождении области нагрева через зону деформации.

На основе полученных экспериментальных данных предложены метод и устройство контроля однородности физико-механических свойств протяженных изделий из сплава с памятью формы, в исходном состоянии находящихся в мартенситной фазе. Предложенные способ и устройства относятся к методам неразрушающего контроля требуемых термомеханических характеристик. Предлагаемое техническое решение может быть использовано для контроля не только изделий из сплава с памятью формы, но и других сплавов, испытывающих в зоне нагрева фазовое (аллотропное) превращение.

\section{Литература/References}

1. V.E. Gunter et al. Medical materials and implants with shape memory effect. Tomsk, Tom. un-ty publ. (1998) 487 p. (in Russian) [В.Э. Гюнтер и др. Медицинские материалы и имплантаты с памятью формы. Томск, изд-во Том. ун-та (1998) 487 с.]

2. K. Otsuka, K. Shimizu, Y. Suzuki et al. Alloys with shape memory effect. Moscow, Metallurgiya (1990) 224 p. (in Russian) [К. Ооцука, К. Симидзу, Ю. Судзуки и др. Сплавы с эффектом памяти формы. Москва, Металлургия (1990) 224 с.]

3. Z. G. Wei, R. Sandström, S. Miyazaki. Journal of Materials Science. 33, 3743 (1998). Crossref

4. D. Mantovani. JOM. 52 (10), 36 (2000). Crossref

5. V. Brailovski, S. Prokoshkin, P. Terriault, F. Trochu. Shape memory alloys: fundamentals, modeling and applications. Montreal, ETS Publ. (2003) 844 p.

6. V.E. Gunter et al. Titanium nickelide. Medical material of new generation. Tomsk, MIZ (2006) 296 p. (in Russian) [В.Э. Гюнтер и др. Никелид титана. Медицинский материал нового поколения. Томск, МИЦ (2006) 296 с.]

7. V.V. Rubanik, V.V. Rubanik Jr., O.A. Petrova-Burkina. Materials, technologies, tools. 17 (1), 25 (2012). (in Russian) [В.В. Рубаник, В.В. Рубаник мл., О.А. Петрова-Буркина. Материалы, технологии, инструменты. 17 (1), 25 (2012).]

8. V.V. Rubanik, V.V. Rubanik Jr., O.A. Petrova-Burkina. Materials of the 9th European Symposium on Martensitic Transformations «ESOMAT 2012». Saint-Petersberg (2012), p. 40.

9. V.V. Rubanik, V.V. Rubanik Jr, O.A. Petrova-Burkina. Materials Science Forum. 738-739, 292 (2013). Crossref

10. V. V. Rubanik, V. V. Rubanik Jr, O.A. Petrova-Burkina. Shape Memory \& Superelastic Technology (SMST 2019). Konstanz, Germany (2019) p. 86.

11. V. V. Rubanik, V.V. Rubanik Jr., O.A. Petrova-Burkina. Letters on Materials. 2 (2), 71 (2012). (in Russian) [В.В. Рубаник, В.В. Рубаник мл., О.А. ПетроваБуркина. Письма о материалах. 2 (2), 71 (2012).] Crossref

12. Standard Test Method for Transformation Temperature of Nickel-Titanium Alloys by Thermal Analysis: ASTM F2004-00, ASTM, 100 BarrHarbor Drive, West Conshohocken, PA, 19428.

13. J.E. Hanlon, S. R. Butler, R. J. Wasilewski. Trans. Met. Soc. AIME. 239, 1323 (1967).

14. A.S. Karolik. Proceed. Intern. Conf. computer methods and inverse problems in nondestructive testing and diagnostics, Belarus, Minsk (1995) p. 210.

15. V. V. Rubanik, A. V. Lesota, V.V. Rubanik jr. Letters on materials. 7 (2), 96 (2017). (in Russian) [В.В. Рубаник, А. В. Лесота, В. В. Рубаник мл. Письма о материалах. 7 (2), 96 (2017).] Crossref

16. A. V. Lesota, V.V. Rubanik, V.V. Rubanik Jr. Letters on Materials. 8 (4), 401 (2018). Crossref

17. Patent BY № 19012, 28.02.2015. (in Russian) [Патент РБ № № 19012, 28.02.2015.]

18. Patent BY № 19017, 28.02.2015. (in Russian) [Патент РБ № № 19017, 28.02.2015.] 